\title{
THE PROBLEM OF FIRST INTEGRAL OF NONHOLONOMIC SYSTEMS
}

\author{
Do SANH \\ Hanoi University of Technology
}

\begin{abstract}
In this paper the problem of first integrals of a nonholonomic system is discussed. The aim of this work is concentrated on finding the condition for existence of first integrals. The obtained results are applied for the construction of linear and quadratic integrals of nonholonomic systems. It obtains two important affirmations; they are: Any first integral could be treated as a particular nonholonomic constraint and contrarily, any nonholonomic constraint could be regarded as a first integral of the nonholonomic system.
\end{abstract}

\section{Introduction}

The construction of first integrals of nonholonomic systems is one of the hardest problems of dynamics of nonholonomic systems, due to the presence of nonholonomic constraints. There is also no convenient form of equations of motion that describe these systems.

It is known presently the equations of motion applied for nonholonomic systems are either the Lagrange's or the Appell's equations.

In the first case the unknowns are increased due to introducing multipliers, but in the latter, the calculation of the acceleration energy is difficult. Investigations about this field have been done very few.

In order to overcome the above mentioned restrictions, the form of equations of motion for a nonholonomic systems presented in [8] is applied. The form of these equations is analogous to one equation which describes the motion of a nonconservative holonomic system.

2. The condition of existence of a first integral for nonholonomic systems

Let us consider a dynamic system, which is described by the equations

$$
x_{i}=g_{i}\left(x_{1}, x_{2}, \ldots, x_{n}\right),
$$

where $x_{i}$ are the state variables, but $\dot{x}_{i} \equiv \frac{d x_{i}}{d t}$.

As known (see, for example [9], [17]) in order the relation

$$
f\left(x_{1}, x_{2}, \ldots, x_{n}\right)=C=\text { const }
$$


is a first integral of (2.1) the necessary and sufficient condition is

$$
\sum_{i=1}^{N} \frac{\partial f}{\partial x_{i}} g\left(x_{1}, x_{2}, \ldots, x_{n}\right) \stackrel{x_{i}}{=} 0 .
$$

In connection with this, let us consider a system, the position of which is defined by nonholonomic coordinates $q_{i}(i=\overline{1, n})$.

The kinetic energy of the system is of the form

$$
T=\frac{1}{2} \dot{q}^{T} \mathbf{A} \dot{q},
$$

where $\dot{\mathbf{q}}$ is the $n \times 1$ matrix of generalized velocities, but $\dot{\mathbf{q}}^{T}$ is its transportation, $\mathbf{A}$ is of a positive define quadratic matrix of $n$-order, the elements of which are continuous functions of generalized coordinates.

The generalized force of applied forces corresponding to the coordinate $q_{i}$ is denoted by $Q_{i}(i=\overline{1, n})$.

Suppose that the system under consideration is subjected to the nonholonomic constraints

$$
\sum_{i=1}^{n} b_{\alpha i} \dot{q}_{i}+b_{\alpha}=0, \quad \alpha=\overline{1, s}
$$

which could be written in the matrix form

$$
\mathbf{b} \dot{\mathbf{q}}+\mathbf{b}^{0}=\mathbf{0},
$$

where $\mathbf{b}$ and $\mathbf{b}^{0}$ are the $s \times n$ and $s \times 1$ matrices respectively, the elements of which are continuous functions of coordinates and time.

As shown in [6], the motion of a constrained system could be described by the matrix equation:

$$
A \ddot{q}=\mathbf{Q}+\mathbf{G}+\mathbf{R},
$$

where $\ddot{\mathbf{q}}$ is the $n \times 1$ matrix of generalized accelerations, $\mathbf{Q}$ - the $n \times 1$ matrix of generalized forces, $G$-the $n \times 1$ matrix is made of the matrix of inertia $\mathbf{A}, \mathbf{R}$ is the $n \times 1$ matrix of reaction forces due to the constraints (2.5).

As shown in [6], the reaction forces of the constraints (2.5) are determined by means of the closed system of algebraic equations

$$
\begin{aligned}
& \mathbf{B} \mathbf{R}=\mathbf{B}^{0}, \\
& \mathbf{D} \mathbf{R}=\mathbf{0},
\end{aligned}
$$

where $\mathbf{B}$ and $\mathbf{B}^{0}$ are the $s \times n$ and $s \times 1$ matrices respectively, that are

$$
\mathbf{B}=\mathbf{b} \mathbf{A}^{*} ; \quad \mathbf{B}^{0}=-\mathbf{A}^{*}(\mathbf{Q}+\mathbf{G})+\dot{\mathbf{b}} \dot{\mathbf{q}}+\dot{\mathbf{b}}^{0}
$$


$\mathrm{D}$ is the $k \times n$ matrix, the elements of which are coefficients in expressions of generalized accelerations represented in terms of $k=n-s$ pseudo-accelerations [6]. $\mathbf{A}^{*}$ is the inverse of the matrix of inertia $\mathbf{A}$, but $\dot{\mathbf{b}}$ and $\dot{\mathbf{b}}^{0}$ are the derivative matrices, the elements of which are the derivations of elements of the matrices $\mathbf{b}$ and $\mathbf{b}^{0}$ respectively.

Solving the equations (2.8) and (2.9) we get

$$
\therefore \mathbf{R}=\mathbf{R}(t, \dot{\mathbf{q}}, \mathbf{q})
$$

which plays the part of additional nonconservative forces effecting on the released system.

Starting with this point of view, it is possible to apply the method of construction of first integrals for holonomic systems to nonholonomic onces [9].

Suppose that the nonholonomic system (2.7) possesses a first integral of the form

$$
f\left(q_{1}, q_{2}, \ldots, q_{n}, \dot{q}_{1}, \dot{q}_{2}, \ldots, \dot{q}_{n}\right)=\text { const. }
$$

By following (2.3) the relation (2.12) will be a first integral of the system (2.7) if and only if

$$
\left[\mathbf{f}_{\mathbf{q}} \dot{\mathbf{q}}+f_{\dot{\mathbf{q}}} \mathbf{A}^{*}(\mathbf{Q}+\mathbf{G}+\mathbf{R})\right]_{(2.5)} \equiv 0,
$$

where $f_{\mathbf{q}}$ and $f_{\dot{q}}$ are the $1 \times n$ matrices of the form

$$
\begin{aligned}
& f_{\mathbf{q}}=\left\|\frac{\partial f}{\partial q_{1}} \frac{\partial f}{\partial q_{2}} \cdots \frac{\partial f}{\partial q_{n}}\right\| \\
& f_{\dot{q}}=\left\|\frac{\partial f}{\partial \dot{q}_{1}} \frac{\partial f}{\partial \dot{q}_{2}} \cdots \frac{\partial f}{\partial \dot{q}_{n}}\right\| .
\end{aligned}
$$

The notion [ ] $]_{(2.5)}$ means that the identify (2.13) is satisfied in the condition of realizing the relations (2.5), i.e. in the existence of effect of the nonholonomic constraints.

Thus, the condition of existence of a first integral of a nonholonomic system is established. In order to write the condition (2.13), it is necessary to compute the reaction forces of nonholonomic constraints. It is required to write and to solve the system of algebraic equations (2.8) and (2.9). Clearly, in some cases such a manner is not always comfortable, especially for the systems with great number of degrees of freedom.

We now will consider another way. As known [7] the motion of a nonholonomic system could be written in the following form

$$
\mathbf{D}^{0} \ddot{\mathrm{q}}=\mathbf{Q}^{0},
$$

where $\mathbf{D}^{0}$ and $\mathbf{Q}^{0}$ are the $k \times n$ and $k \times 1$ matrices respectively, that are

$$
\begin{aligned}
& \mathbf{D}^{0}=\mathbf{D} \mathbf{A}, \\
& \mathbf{Q}^{0}=\mathbf{D}(\mathbf{Q}+\mathbf{G}) .
\end{aligned}
$$


The equation (2.16) together the equation (2.6) describe the motion of the system under consideration.

By means of (2.16) and (2.6) it is possible to compute the generalized accelerations, that is

$$
\ddot{\mathrm{q}}=\mathrm{S}^{*} \mathbf{Q}^{*},
$$

where $\mathbf{S}^{*}$ is the $n \times n$ matrix, which is the inverse of the $n \times n$ matrix $\mathbf{S}$

$$
\mathrm{S}=\left\|\begin{array}{c}
\mathrm{D}^{0} \\
\mathbf{b}
\end{array}\right\|
$$

but $\mathrm{Q}^{*}$ is the $n \times 1$ matrix of the form

$$
\mathbf{Q}^{*}=\left\|\begin{array}{l}
\mathbf{Q}^{0} \\
\mathbf{b}^{*}
\end{array}\right\|
$$

in which the $s \times 1$ matrix $\mathrm{b}^{*}$ will be

$$
\mathbf{b}^{*}=-\left(\dot{\mathbf{b}}^{0}+\dot{\mathbf{b}} \dot{\mathbf{q}}\right) .
$$

The condition (2.13) takes the form now

$$
\left[f_{\mathbf{q}} \dot{\mathbf{q}}+f_{\dot{\mathbf{q}}} \mathbf{S}^{*} \dot{\mathbf{Q}}^{*}\right]_{(2.5)} \stackrel{\mathrm{q}, \dot{\mathbf{q}}}{=} 0
$$

From the above investigation, two important affirmations are obtained.

Affirmation 1. The equations of nonholonomic constraints could be regarded as first integrals of the equations of motion of the system under consideration.

It is clear, because the equations (2.16) and (2.6) are equivalent to any of the equations described by the motion of the nonholonomic system obtained by only some elementary transformations.

The presence of (2.5) in a complete set of equations of motion of the nonholonomic system proves that, the equations of constraints could be treated as first integrals.

Affirmation 2. The first integrals could be treated as additional constraints subjected to the system under consideration with the assumption that their reaction forces are equal to zero.

This means that the additional constraints do not cause any dynamic effect upon the given system. In order to prove this, the equations of motion of the nonholonomic system without additional constraints are written in the form:

$$
\mathbf{A} \ddot{\mathbf{q}}=\mathbf{Q}+\mathbf{G}+\mathbf{R},
$$


where $\mathbf{R}$ is the matrix of reaction forces of nonholonomic constraints, which are known functions of velocities, coordinates and time. In the present of additional constraint (2.12), by the Principle of Compatibility the equations of motion of the system are:

$$
A \ddot{q}=Q+G+R+R^{*}
$$

where $\mathbf{R}^{*}$ is the matrix of reaction forces of the additional constraints (2.12), which is computed by a closed set of algebraic equations

$$
\begin{aligned}
& \mathbf{F} \mathbf{R}^{*}+\mathbf{f}_{\dot{\mathbf{q}}} \mathbf{A}^{*}(\mathbf{Q}+\mathbf{G}+\mathbf{R})+\mathbf{f}_{\mathbf{q}} \dot{\mathbf{q}}=0, \\
& \mathbf{D}^{*} \mathbf{R}^{*}=0,
\end{aligned}
$$

where $\mathbf{F}$ is the $s \times n$ matrix

$$
\mathbf{F}=\mathbf{f}_{\mathbf{q}} \mathbf{A}^{*}
$$

but $\mathbf{D}^{*}$ is $(n-s) \times n$ matrix, which is defined in same way as $\mathbf{D}$.

It is easy to observe that

$$
\operatorname{rank}\left\|\begin{array}{c}
\mathbf{F} \\
\mathbf{D}^{*}
\end{array}\right\|=n .
$$

Because (2.12) is the first integral then we have (2.13). Whence

$$
\begin{aligned}
& \mathbf{F} \mathbf{R}^{*}=0 \\
& \mathbf{D}^{*} \mathbf{R}^{*}=0
\end{aligned}
$$

By (2.28) it draws immediately

$$
\mathbf{R}^{*}=0
$$

\section{Commentary}

1. By Affirmation 1 it is possible to obtain another integral by eliminating dependent velocities from the given integral with the help of nonholonomic constraint equations. This is due to a combination of first integrals, which also is a first integral. This could lessen the integration of equations of motion of the system under consideration.

2. The assumption of first integrals to be nonholonomic constraints subjected to the system considered, allows to apply the method of nonholonomic mechanics to the mechanical system controlled by programmes. By means of the affirmation 2, it could regard the programmes as constraints in analytical mechanics.

\section{The condition of existence of linear and quadratic integrals}

First let us consider the case of linear integrals with respect to velocities which are called briefly linear integrals. 
Suppose that the system with the nonholonomic constraints (2.5) possesses a first integral of the form

$$
\xi \dot{\mathrm{q}}+\xi^{0}=\text { const, }
$$

where $\xi$ is the $1 \times n$ matrix, the elements of which together the function $\xi^{0}$ are continuous onces of generalized coordinates, i.e.

$$
\xi=\left\|\xi_{1}, \xi_{2}, \ldots, \xi_{n}\right\|
$$

and

$$
\xi_{i}=\xi_{i}\left(q_{j}\right), \quad \xi^{0}=\xi^{0}\left(q_{j}\right) .
$$

The condition (2.23) now takes the form

$$
\left[\xi S^{*} Q^{*}+\dot{\xi} \dot{\mathbf{q}}+\xi_{q}^{0} \dot{\mathbf{q}}\right]_{(2.6)} \stackrel{\underline{\dot{q}}}{=} 0,
$$

where the $1 \times n$ matrix $\dot{\xi}$ consists of the elements to be the derivations of the ones of the matrix $\boldsymbol{\xi}$ and $\boldsymbol{\xi}_{q}^{0}$ is the $1 \times n$ matrix, that are

$$
\begin{aligned}
\dot{\boldsymbol{\xi}} & =\left\|\dot{\xi}_{1}, \dot{\xi}_{2}, \ldots, \dot{\xi}_{n}\right\|, \\
\boldsymbol{\xi}_{q}^{0} & =\left\|\frac{\partial \xi^{0}}{\partial q_{1}} \frac{\partial \xi^{0}}{\partial q_{2}} \ldots \frac{\partial \xi^{0}}{\partial q_{n}}\right\| .
\end{aligned}
$$

Besides, it is easy to observe that

$$
\dot{\xi} \dot{\mathrm{q}}=\sum_{i, j=1}^{n} \frac{\partial \xi_{i}}{\partial q_{j}} \dot{q}_{i} \dot{q}_{j}
$$

By identifying (3.3) with respect to generalized velocities we obtain a set of partial differential equations, which allow to define the unknowns $\xi_{i}(i=\overline{1, n})$ and $\xi^{0}$

Example 1. For illustration let us consider the inertia motion in the horizontal plane of the Chaplyghin's sledge $[4,8,15]$.

The kinetic energy of the system is of the form

$$
T=\frac{1}{2} m\left(\dot{x}^{2}+\dot{y}^{2}\right)+\frac{1}{2} m k^{2} \dot{\varphi}^{2}
$$

where $m$ is the mass, but $k$ - the inertia radius of the sledge about the mass centre, the projection of which in the horizontal plane is coincided with the contact point between the wheel and plane (Fig. 1). The $x, y$ are the coordinates of the mass centre of the sledge, but $\varphi$ is the angle between the line of intersection of the wheel plane and the horizontal plane with respect to the $x$ 's axis.

As shown $[4,15]$ the motion without sliding of the slegde is restricted to the nonholonomic constraints 


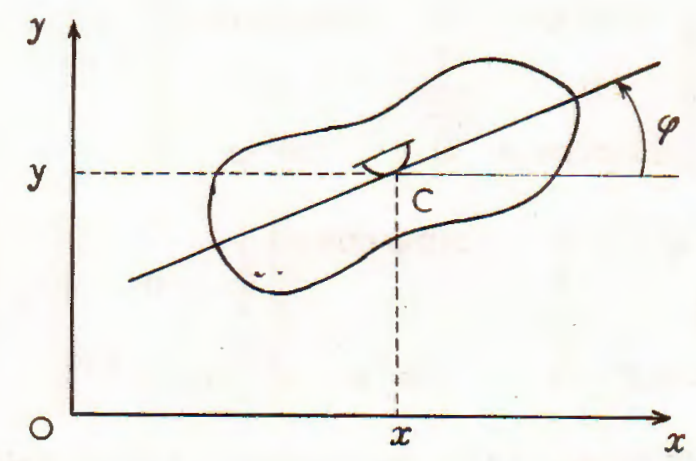

Fig. 1

$$
\dot{y}-\dot{x} \operatorname{tg} \varphi=0 .
$$

The matrix $\mathbf{D}$ now takes the form

$$
\mathrm{D}=\left\|\begin{array}{ccc}
1 & 0 & \operatorname{tg} \varphi \\
0 & 1 & 0
\end{array}\right\| .
$$

It is easy to compute the following matrices

$$
\begin{aligned}
& \mathbf{b}=\|-\operatorname{tg} \varphi \quad 0 \quad 1\|, \\
& \mathbf{b}^{*}=-\|\left(\dot{\mathbf{b}}+\dot{\mathbf{b}} \dot{\mathbf{q}}\|=\| \frac{1}{\cos ^{2} \varphi} \dot{\varphi} \quad 0 \quad 0\|\|\left\|\begin{array}{l}
\dot{x} \\
\dot{\varphi}
\end{array}\right\|=\frac{1}{\cos ^{2} \varphi} \dot{x} \dot{\varphi},\right. \\
& \mathbf{D}^{0}=\mathbf{D} \mathbf{A}=\left\|\begin{array}{ccc}
1 & 0 & \operatorname{tg} \varphi \\
0 & 1 & 0
\end{array}\right\|\left\|\begin{array}{ccc}
m & 0 & 0 \\
0 & m k^{2} & 0 \\
0 & 0 & m
\end{array}\right\|=\left\|\begin{array}{ccc}
m & 0 & m \operatorname{tg} \varphi \\
0 & m k^{2} & 0
\end{array}\right\| \text {, } \\
& \mathbf{Q}^{0}=\mathbf{D} \mathbf{Q}=\left\|\begin{array}{ccc}
1 & 0 & \operatorname{tg} \varphi \\
0 & 1 & 0
\end{array}\right\|\left\|\begin{array}{l}
0 \\
0 \\
0
\end{array}\right\|=\left\|\begin{array}{l}
0 \\
0
\end{array}\right\| \text {, } \\
& \mathbf{Q}^{*}=\left\|\begin{array}{c}
\mathbf{Q}^{0} \\
\mathbf{b}
\end{array}\right\|=\left\|\begin{array}{c}
0 \\
0 \\
\frac{1}{\cos ^{2} \varphi} \dot{x} \dot{\varphi}
\end{array}\right\| \\
& \mathbf{S}=\left\|\begin{array}{c}
\mathbf{D}^{0} \\
\mathbf{b}
\end{array}\right\|=\left\|\begin{array}{ccc}
m & 0 & m \operatorname{tg} \varphi \\
0 & m k^{2} & 0 \\
-\operatorname{tg} \varphi & 0 & 1
\end{array}\right\|
\end{aligned}
$$




$$
\begin{aligned}
\mathbf{S}^{*} & =\mathbf{S}^{-1}=\left\|\begin{array}{ccc}
\cos ^{2} \varphi & 0 & -\sin \varphi \cos \varphi \\
0 & \frac{1}{k^{2}} & 0 \\
\sin \varphi \cos \varphi & 0 & \cos ^{2} \varphi
\end{array}\right\|, \\
\mathbf{S}^{*} \mathbf{Q}^{*} & =\left\|\begin{array}{ccc}
\cos ^{2} \varphi & 0 & -\sin \varphi \cos \varphi \\
0 & \frac{1}{k^{2}} & 0 \\
\sin \varphi \cos \varphi & 0 & \cos ^{2} \varphi
\end{array}\right\|\left\|\begin{array}{c}
0 \\
0 \\
\frac{1}{\cos ^{2} \varphi} \dot{x} \dot{\varphi}
\end{array}\right\|=\left\|\begin{array}{c}
-\operatorname{tg} \varphi \dot{x} \dot{\varphi} \\
0 \\
\dot{x} \dot{\varphi}
\end{array}\right\| .
\end{aligned}
$$
form

In consideration of (3.6) and (3.7) the identify (3.3) is written in the following

$$
\begin{aligned}
{\left[\xi \mathbf{S}^{*} \mathbf{Q}^{*}+\dot{\boldsymbol{\xi}} \dot{\mathbf{q}}+\xi_{q}^{0} \dot{q}\right]_{(3.7)}=} & \dot{x}^{2}\left[\frac{\partial \xi_{1}}{\partial x}+\left(\frac{\partial \xi_{2}}{\partial y}+\frac{\partial \xi_{3}}{\partial \varphi}\right) \operatorname{tg} \varphi+\frac{\partial \xi_{3}}{\partial y} \operatorname{tg}^{2} \varphi\right] \\
& +\dot{x} \dot{\varphi}\left[\xi_{3}-\xi_{1} \operatorname{tg} \varphi+\frac{\partial \xi_{1}}{\partial \varphi}+\frac{\partial \xi_{2}}{\partial x}+\left(\frac{\partial \xi_{2}}{\partial y}+\frac{\partial \xi_{3}}{\partial \varphi}\right) \operatorname{tg} \varphi\right] \\
& +\dot{\varphi}^{2} \frac{\partial \xi_{2}}{\partial \varphi}+\dot{x}\left(\frac{\partial \xi^{0}}{\partial y} \operatorname{tg} \varphi+\frac{\partial \xi^{0}}{\partial x}\right)+\dot{\varphi} \frac{\partial \xi^{0}}{\partial \varphi} \stackrel{\dot{x}, \dot{\varphi}}{=} 0
\end{aligned}
$$

By identifying the obtained equality with respect to velocities, we get

$$
\begin{aligned}
& \frac{\partial \xi_{1}}{\partial x}+\left(\frac{\partial \xi_{1}}{\partial y}+\frac{\partial \xi_{3}}{\partial x}\right) \operatorname{tg} \varphi+\frac{\partial \xi_{3}}{\partial y} \operatorname{tg}^{2} \varphi=0, \\
& \frac{\partial \xi_{1}}{\partial \varphi}+\frac{\partial \xi_{2}}{\partial x}+\left(\frac{\partial \xi_{2}}{\partial y}+\frac{\partial \xi_{3}}{\partial \varphi}\right) \operatorname{tg} \varphi+\xi_{3}-\xi_{1} \operatorname{tg} \varphi=0, \\
& \frac{\partial \xi_{2}}{\partial \varphi}=0, \quad \frac{\partial \xi^{0}}{\partial \varphi}=0, \quad \frac{\partial \xi^{0}}{\partial x}+\frac{\partial \xi^{0}}{\partial y} \operatorname{tg} \varphi=0 .
\end{aligned}
$$

These equations possesse particular solutions of the form First solution

$$
\xi_{1}=\frac{1}{\cos \varphi}, \quad \xi_{2}=0, \quad \xi_{3}=0, \quad \xi^{0}=0,
$$

which corresponds with the first integral

$$
\frac{\dot{x}}{\cos \varphi}=C_{1}
$$

Second solution

$$
\xi_{1}=\frac{1}{\cos \varphi}, \quad \xi_{2}=0 . \quad \xi_{3}=\frac{1}{\sin \varphi}, \quad \xi^{0}=0
$$


which gives the first integral

$$
\frac{\dot{x}}{\cos \varphi}+\frac{\dot{y}}{\sin \varphi}=C_{2} .
$$

Let us now consider the existence of quadratic integrals.

Suppose that the system with nonholonomic constraints (2.6) possesses a first integral of the form

$$
\phi=\frac{1}{2} \dot{\mathrm{q}}^{T} \theta \dot{\mathrm{q}}=\text { const },
$$

where $\theta$ is a $n$-order quadratic matrix, the elements of which are continuous functions of generalized coordinates.

The conditions of existence of the integral (3.1) by following (2.23) will be

$$
\left[\frac{1}{2} \mathbf{Q}^{*} \mathbf{S}^{*} \theta \dot{\mathbf{q}}+\frac{1}{2} \dot{\mathbf{q}}^{T} \theta \mathbf{S}^{*} \mathbf{Q}+\frac{1}{2} \dot{\mathbf{q}}^{T} \dot{\boldsymbol{\theta}} \dot{\mathbf{q}}\right]_{(2.5)} \stackrel{\dot{\mathrm{q}}}{=} 0 \text {. }
$$

In the case of symmetric matrix $\theta$, we have

$$
\left[2 \dot{\mathbf{q}}^{T} \theta \mathbf{S}^{*} \mathbf{Q}^{*}+\dot{\mathrm{q}}^{T} \dot{\theta} \dot{\mathbf{q}}\right]_{(2.5)} \stackrel{\dot{\dot{q}}}{=} 0 \text {. }
$$

Observe that the last term in the left member of (3.10) is of the form

$$
\dot{\mathbf{q}}^{T} \dot{\boldsymbol{\theta}} \dot{\mathrm{q}}=\sum_{i, j, \sigma=1}^{n} \frac{\partial \theta_{i j}}{\partial q_{\sigma}} \dot{q}_{i} \dot{q}_{j} \dot{q}_{\sigma}
$$

For illustration let us return to Example 1.

Suppose that the motion of the Chaplyghin's sledge subjected to the nonholonomic constraint (3.7) possesses a first integral of the form

$$
\phi=\frac{1}{2}\left(\theta_{11} \dot{x}^{2}+\theta_{22} \dot{\varphi}^{2}+\theta_{33} \dot{y}^{2}+2 \theta_{12} \dot{x} \dot{\varphi}+2 \theta_{13} \dot{x} \dot{y}+2 \theta_{23} \dot{\varphi} \dot{y}\right) .
$$

The identify (3.10) in consideration of (3.11), (3.7) and the results obtained in Example 1 will be

$$
\begin{aligned}
& \dot{x}^{3}\left[\frac{\partial \theta_{11}}{\partial x}+\left(\frac{\partial \theta_{11}}{\partial y}+2 \frac{\partial \theta_{13}}{\partial x}\right) \operatorname{tg} \varphi+\left(\frac{\partial \theta_{12}}{\partial x}+2 \frac{\partial \theta_{13}}{\partial x}+2 \frac{\partial \theta_{13}}{\partial y}\right) \operatorname{tg} \varphi+\right. \\
& \dot{x}^{2} \dot{\varphi}\left[\frac{\partial \theta_{11}}{\partial \varphi}+2 \frac{\partial \theta_{12}}{\partial x}+2\left(\frac{\partial \theta_{12}}{\partial y}+\frac{\partial \theta_{23}}{\partial x}+\frac{\partial \theta_{23}}{\partial y}+\frac{\partial \theta_{13}}{\partial \varphi}\right) \operatorname{tg} \varphi+\right. \\
& \left.\quad \frac{\partial \theta_{33}}{\partial \varphi} \operatorname{tg}^{2} \varphi+2 \theta_{13}+2\left(\theta_{33}-\theta_{11}\right)-2 \theta_{13} \operatorname{tg}^{2} \varphi\right]+ \\
& \dot{x} \dot{\varphi}\left[\frac{\partial \theta_{22}}{\partial x}+2 \frac{\partial \theta_{12}}{\partial \varphi}+\left(\frac{\partial \theta_{22}}{\partial y}+2 \frac{\partial \theta_{23}}{\partial \varphi}\right) \operatorname{tg} \varphi+2\left(\theta_{23}-\theta_{12} \operatorname{tg} \varphi\right)\right]+\dot{\delta}^{3} \frac{\partial \theta_{22}}{\partial \varphi} \stackrel{\dot{x} \dot{\varphi}}{=} 0 .
\end{aligned}
$$


Hence we get:

$$
\begin{aligned}
& \frac{\partial \theta_{11}}{\partial x}+\left(\frac{\partial \theta_{11}}{\partial y}+2 \frac{\partial \theta_{13}}{\partial x}\right) \operatorname{tg} \varphi+\left(\frac{\partial \theta_{33}}{\partial x}+2 \frac{\partial \theta_{13}}{\partial y}\right) \operatorname{tg}^{2} \varphi+\frac{\partial \theta_{33}}{\partial y} \operatorname{tg}^{3} \varphi=0 \\
& \frac{\partial \theta_{11}}{\partial \varphi}+2 \frac{\partial \theta_{12}}{\partial x}+2\left(\frac{\partial \theta_{12}}{\partial y}+\frac{\partial \theta_{23}}{\partial x}+\frac{\partial \theta_{23}}{\partial y}+\frac{\partial \theta_{13}}{\partial \varphi}+\theta_{33}-\theta_{11}\right) \operatorname{tg} \varphi \\
& \quad+\left(\frac{\partial \theta_{33}}{\partial \varphi}-2 \theta_{13}\right) \operatorname{tg}^{2} \varphi+2 \theta_{13}=0 \\
& \frac{\partial \theta_{22}}{\partial x}+2 \frac{\partial \theta_{12}}{\partial \varphi}+\left(\frac{\partial \theta_{22}}{\partial y}+2 \frac{\partial \theta_{23}}{\partial \varphi}-2 \theta_{12}\right) \operatorname{tg} \varphi+2 \theta_{23}=0 \\
& \frac{\partial \theta_{22}}{\partial \varphi}=0
\end{aligned}
$$

Observe that these equations have particular solutions

$$
\theta_{11}=\frac{1}{\cos ^{2} \varphi} ; \quad \theta_{13}=\frac{1}{\sin \varphi \cos \varphi} ; \quad \theta_{22}=\theta_{33}=\theta_{23}=\theta_{12}=0
$$

which corresponds with the first integral

$$
\frac{\dot{x}^{2}}{\cos ^{2} \varphi}-\frac{\dot{x} \dot{y}}{\sin \varphi \cos \varphi}=\text { const. }
$$

It is easy to show that the integral obtained is a combination of linear integrals found in Example 1.

Second solution

$$
\theta_{11}=\theta_{33}=C_{1}, \quad \theta_{22}=C_{2}, \quad \theta_{12}=\theta_{13}=\theta_{34}=0,
$$

by which we obtain the integral

$$
C_{1}\left(\dot{x}^{2}+\dot{y}^{2}\right)+C_{2} \dot{\varphi}^{2}=\text { const. }
$$

In the case of $C_{1}=m, C_{2}=m k^{2}$ we have the integral of energy.

4. The existence of the integral of energy and the generalized integral of energy

First let us consider a conservative system, the position of which is defined by holonomic coordinates $q_{i}(i=\overline{1, n})$, but the kinetic energy is of the form (2.4) and the potential energy $\Pi$ is a continuous function of generalized coordinates i.e.

$$
\Pi=\Pi\left(q_{1}, q_{2}, \ldots, q_{n}\right) .
$$

Suppose that system is subjected to the nonholonomic constraints of the form

$$
\mathbf{b} \dot{\mathbf{q}}=\mathbf{0} \text {. }
$$


It is easy to show that for the system under consideration the integral of energy always exists

$$
E=T\left(q_{1}, q_{2}, \ldots, q_{n}, \dot{q}_{1}, \dot{q}_{2}, \ldots, \dot{q}_{n}\right)+\Pi\left(q_{1}, q_{2}, \ldots, q_{n}\right)=\text { const. }
$$

Indeed, the motion of the system could be described by the Lagrange's equations with multipliers

$$
\frac{d}{d t} \frac{\partial T}{\partial \dot{\mathbf{q}}}-\frac{\partial T}{\partial \mathbf{q}}=-\frac{\partial \Pi}{\partial \mathbf{q}}+\mathbf{b}^{T} \Lambda
$$

where $\Lambda$ is a $s \times 1$ matrix of the Lagrange's multipliers $\Lambda_{\alpha}(\alpha=\overline{1, s})$, but the letter $T$ located on the hight right corner denotes the transportation.

Observe, the following identify

$$
\frac{d}{d t}(T+\Pi)=\left(\frac{d}{d t} \frac{\partial T}{\partial \dot{\mathbf{q}}}-\frac{\partial T}{\partial \mathbf{q}}+\frac{\partial \Pi}{\partial \mathbf{q}}\right)^{T} \dot{\mathbf{q}}
$$

In consideration of (4.4) and (4.5) we have

$$
\frac{d}{d t}(T+\Pi)=\mathbf{\Lambda}^{T} \mathbf{b} \dot{\mathbf{q}}
$$

Due to (4.2), we obtain

$$
\frac{d}{d t}(T+\Pi)=0
$$

The existence of the integral of energy (4.3) is then affirmed.

The result obtained proves that the reaction forces of the constraints of the form (4.2) belong to the kind of gyroscope forces.

In the case where nonholonomic constraints are of the form (2.6), the condition of the existence of the integral of energy (4.3) in consideration (3.10) will be of the form

$$
\left[2 \dot{\mathbf{q}}^{T} \mathbf{A ~ S}^{*} \mathbf{Q}^{*}+\dot{\mathbf{q}}^{T} \dot{\mathbf{A}} \dot{\mathbf{q}}+\frac{\partial \Pi}{\partial \mathbf{q}} \dot{\mathbf{q}}\right]_{(2.6)} \stackrel{\underline{\dot{q}}}{=} 0 \text {. }
$$

The second term in the left member of (4.8) is calculated as follows

$$
\dot{\mathbf{q}}^{T} \dot{\mathbf{A}} \dot{\mathbf{q}}=\sum_{i, j, \sigma=1}^{n} \frac{\partial a_{i j}}{\partial q_{\sigma}} \dot{q}_{i} \dot{q}_{j} \dot{q}_{\sigma},
$$

but $a_{i j}=a_{i j}\left(\dot{q}_{1}, q_{2}, \ldots, q_{n}\right)$ are elements of the matrix of inertia.

Let us now consider the case of the generalized integral of energy.

It is known that the generalized integral of energy is constructed by eliminating dependent velocities from the expression of the mechanical energy $E=T+\Pi$ 
with the help of the constraint equations (2.5). In [15], this problem has been demonstrated in a complicated manner.

By means of the affirmation obtained in the paragraph 2, it is possible to obtain immediately.

The generalized integral of energy also is a integral of the equations of motion of the nonholonomic system under consideration.

For illustration let us turn back to Example 1. The motion of the sledge is described by means of equations (2.16) and (2.5), which are

$$
\left\|\begin{array}{ccc}
m & 0 & m \operatorname{tg} \varphi \\
0 & m k^{2} & 0
\end{array}\right\|\left\|\begin{array}{c}
\ddot{x} \\
\ddot{\varphi} \\
\ddot{y}
\end{array}\right\|=0
$$

In the expanded form we have

$$
\begin{aligned}
\ddot{x}+\ddot{y} \operatorname{tg} \varphi & =0, \\
\ddot{\varphi} & =0, \\
\dot{y}-\dot{x} \operatorname{tg} \varphi & =0,
\end{aligned}
$$

which could be written in the equivalent form

$$
\begin{aligned}
& \ddot{x}=-\operatorname{tg} \varphi \dot{x} \dot{\varphi}, \\
& \ddot{\varphi}=0, \\
& \ddot{y}=\dot{x} \dot{\varphi} .
\end{aligned}
$$

As known, there exists the integral of energy

$$
E=T+\Pi=\frac{1}{2} m\left(\dot{x}^{2}+\dot{y}^{2}\right)+\frac{1}{2} m k^{2} \dot{\varphi}^{2}=\text { const. }
$$

By eliminating the dependent velocity $\dot{y}$ from the expression $E$ with the help of the constraint equation (3.7) we have

$$
E^{*}(x, \varphi, y, \dot{x}, \dot{\varphi})=\frac{1}{2} m\left(\frac{1}{\cos ^{2} \varphi} \dot{x}^{2}+k^{2} \dot{\varphi}^{2}\right) .
$$

Clearly. the equations (4.12) possesse an integral of the form

$$
\frac{1}{2} m\left(\frac{1}{\cos ^{2} \varphi} \dot{x}^{2}+k^{2} \dot{\varphi}^{2}\right)=\text { const, }
$$

which is called the generalized integral of energy.

This is evident, because

$$
\frac{d E^{*}}{d t}=m\left[\frac{\dot{x} \ddot{x}}{\cos ^{2} \varphi}+\frac{\operatorname{tg} \varphi}{\cos ^{2} \varphi} \dot{x}^{2} \dot{\varphi}+k^{2} \dot{\varphi} \ddot{\varphi}\right]_{\substack{\ddot{x}=-\operatorname{tg} \varphi \dot{x} \dot{\varphi} \\ \dot{\varphi}=0 \\ \dot{y}=\dot{x} \dot{\varphi}}} \equiv 0
$$


The affirmation of the existence of the generalized integral of energy is very important because the most of nonholonomic constraints take the form of (4.2). Therefore, for the conservative system with the constraints (4.2), the integral of energy always exists. Consequently, the generalized integral of energy of the system under consideration certainly exists. This integral contains only independent velocities. This will decrease the integration of the motion of the system under consideration.

\section{Conclusion}

In this paper, the method for constructing first integrals of a nonholonomic system was introduced. The method obtained was applied for finding linear and quadratic integrals.

This paper demonstrates that the first integral of a nonholonomic constraint can be regarded as a particular nonholonomic constraint possessing zero reaction forces and contrarily the equations of nonholonomic constraints also can be treated as first integrals.

The obtained results play an important role in the investigation of controlled mechanical systems.

This work is completed with financial support of the Council for Natural Science of Vietnam.

\section{REFERENCES}

1. Chetaev N. G. Theory of stability of motion, The Selected Papers on Analytical Mechanics, Publ. USSR Acad, Moscow 1992 (in Russian).

2. Dobronravov V. V. Controlled mechanical system is regarded a mechanical system with nonholonomic constraints, Mechanics, No 104, Publ. Obrongiz, 1961 (in Russian).

3. Dobronravov V. V. Integrals of dynamics equations are regarded nonholonomic constraints, Mechanics, Publ. Obrongiz, 1963 (in Russian).

4. Dobronravov V. V. Fundamental of nonholonomic mechanics, Publ. Vyshaia Shkola, Moscow. 1970 (in Russian).

5. Dobronravov V. V. Fundamental of analytical mechanics, Publ. Vyshaja Shkola, Moscow, 1976 (in Russian).

6. Do Sanh. On the motion of constrained mechanical system, The Thesis of Doctor of Science, Hanoi University of Technology; Hanoi, 1984 (in Vietnamese).

7. Do Sanh. A form of equations of motion of mechanical systems, Journal of Mechanics, NCNST of Vietnam, No 3, 1995 (in Vietnamese). 
8. Do Sanh. A form of equation of motion of a constrained mechanical system, Proceedings of International Conference of Applied of Dynamics, Hanoi, 1995.

9. Do Sanh. On the problem of first integrals of mechanical systems, Proceedings of the National Conference on Engineering Vibrations, 3-5 September, 2000, Hanoi.

10. Erughin N. P. Construction of a set of differential equations possessing a given curse, PMM No 6, 1952 (in Russian).

11. Kirghetov V.I. On the motion of controlled mechanical systems with conditional constraints, PMM No 3, 1967 (in Russian).

12. Korenev G. V. Target and motion by direction, Publ. Nauka, 1974 (in Russian).

13. Muller P. C. Popp K. Zur theorie der ersten integrall bei gestewerten dynamischen system, ZAMM T. 48, T. 59, 1974

14. Muller P. C. Popp K. First integrais of controlled mechanical systems, Gyrodynamics.

15. Neimark Ju, Fufaev N. A. Dynamics of nonholonomic systems, Publ. Nauka, Moscow, 1967 (in Russian).

16. Potsarixkji G. K. Construction of Liapunov's function by equations of pertubation motion, PMM No 2, 1958 (in Russian).

17. Ruraviev V. F. Fundamental of theoretial mechanics, Publ. Nankla, Fizmat., 1997 (in Russian).

Received November 8, 2000

VỀ BÀI TOÁN TÍCH PHÂN ĐẦU CỬ CÁC HỆ KHÔNG HÔLÔNÔM

Trong công trình kháo sát các điều kiện tồn tại các tích phân đầu của các hệ không hô lônom và nhờ đó nhận được phıæøng pháp xây dựng các tích phân tuyến tính và bình phırơng đối với vận tốc.

Từ các kết quả nhận được đã chỉ ra rằng có thể xem các tích phân đầu như là những tích phân đặc biệt có phản lực bằng không và các phương trình liên kết không hôlônôm là các tích phân đầu.

Cũng đã chứng minh rằng khi khử các vận tốc phụ thuộc từ một tích phân chia hệ không hôlônôm nhờ các phương trình của liên kết không hôlônôm ta sẽ nhận dược một tích phân đầu khác. Tì̀ dây tìm được tích phân năng lượng mở rộng của hệ không hôlônôm. 